\title{
Health Care Experiences and Needs of Adolescents in Urban Greece: Implications for Planning Appropriate Health Care
}

\section{Adamandia Xekalaki ${ }^{*}$, Dimitris Papamichail ${ }^{2}$, Polixeni Nikolaidou ${ }^{3}$, Anastasios Papadimitriou ${ }^{3}$ and Takis Panagiotopoulos ${ }^{2}$}

${ }^{1}$ Department of Developmental and Social Pediatrics, Institute of Child Health, "Agia Sophia" Childrens' Hospital, Papadiamadopoulou and Thivon, Athens, Greece ${ }^{2}$ Department of Child Health, National School of Public Health, Athens, Greece

${ }^{3}$ National and Kapodistrian University of Athens, School of Medicine, "Attikon" University Hospital, Athens, Greece

\begin{abstract}
Purpose: This study aims to explore experiences and views of 15 year old adolescents on primary health care services in large cities in Greece, and make policy and practice recommendations.

Methods: A representative sample of 15 year old students, living in large cities of Greece, was selected using stratified cluster sampling. Information was collected using a structured questionnaire.

Results: The questionnaire was completed by 2019 of the 2342 students in the school classes selected (response rate $86 \%$ ). Girls were $54 \%$; $92 \%$ were born in Greece. Half of the students $(50 \%)$ reported having communication problems with the physician(s) they usually visit. Reasons of communication problems included: a/organizational problems, e.g. inadequate consultation time (17\%); b/privacy and confidentiality issues, e.g. not having the opportunity to be alone with the doctor (36\%), lack of confidence that their discussion with the doctor will not be disclosed to their parents (46\%); c/lack of understanding their perspective, e.g. the doctor "is not interested in my problems" $(15 \%)$, he/ she "does not treat me with respect" $(13 \%)$. The gender of the physician is important for about one third of the students of the study sample (girls $48 \%$, boys $23 \%$ ) and girls tend to prefer a female doctor (54\%)
\end{abstract}

Conclusion: Adolescents in this study pointed out that they need doctors who are aware of their way of thinking and problems and can communicate effectively with them; they require more consultation time, continuity of care, confidentiality and privacy. Service provision should be accordingly modified.

Keywords: Adolescent; Communication problems; Confidentiality; Health care; Greece

\section{Introduction}

Appropriate adolescent health care delivery is an important component of policies to improve adolescent health [1-3]. A substantial amount of research has shown that important health issues emerge during adolescence that can affect present health status and have a serious impact on adult life [1-6]. Depression, anxiety and other mental health problems, injuries, infectious diseases and risk factors such as iron deficiency, unsafe sex, alcohol and substance misuse contribute to the emerging disease profile of young people [1,3,4,7-9]. Chronic diseases, such as diabetes and early signs of cardiovascular disease, appearing in teenagers and young adults, may lead to a substantial drop in life expectancy [7]. A systematic analysis of mortality trends in children and young people in the past 50 years has shown that all cause mortality in adolescents and young adults was reduced less than in children [10].

Reported poor health, specific health problems and lack of life satisfaction among adolescents, especially girls, have been consistently found to be associated with unfavourable socioeconomic conditions [1,11-14]. In the mid-2000s, mortality of adolescents and young people (10-24 years old) living in low and middle income countries was found 3.6 times higher than that of those living in high income countries [15].

Improving primary health care services and implementing measures to make them appropriate for young people can allow them engage with their health and result in both short and long term population health gains $[1-3,16,17]$. Kang et al. [18] recommended seven principles to improve access and quality of primary health for youth. These principles include access facilitation, evidence-based practice, youth participation, collaboration, professional development, sustainability and evaluation [18]. Globally many countries fail to put emphasis on the health needs of adolescents $[7,19]$. This is also the case in Greece, where adolescents share the same health facilities with adults or with children, with no special care for their particular needs. In addition, the unfolding effects of the financial crisis have an impact not only on people's income but also on the capacity of the health system to promote population health and manage health services $[20,21]$.

This study aims to explore experiences and views of 15 year old adolescents on primary health care services in large cities in Greece, and make policy and practice recommendations.

\section{Methods}

We selected a random sample of 15 year old students (fourth grade of secondary school) living in large cities of Greece using stratified cluster sampling. Cities with a population of more than 60,000 were included,

${ }^{*}$ Corresponding author: Adamandia Xekalaki, Department of Developmental and Social Pediatrics, Institute of Child Health, "Agia Sophia" Childrens' Hospital, Papadiamadopoulou and Thivon, Athens, Greece; Tel: 2104201165; E-mail: a.xekalaki@gmail.com

Received September 21, 2017; Accepted September 26, 2017; Published October 03, 2017

Citation: Xekalaki A, Papamichail D, Nikolaidou P, Papadimitriou A Panagiotopoulos T (2017) Health Care Experiences and Needs of Adolescents in Urban Greece: Implications for Planning Appropriate Health Care. Health Care Current Reviews 5: 208. doi: 10.4172/2375-4273.1000208

Copyright: @ 2017 Xekalaki A, et al. This is an open-access article distributed under the terms of the Creative Commons Attribution License, which permits unrestricted use, distribution, and reproduction in any medium, provided the original author and source are credited. 
stratified by size. We used three strata: a/Athens, b/Thessaloniki, and c/other cities. In each stratum a number of schools were selected proportionate to stratum size according to the population of 15-19 year old. In each stratum, schools (clusters) were selected by systematic sampling, with a probability of selection proportionate to the school size (depending on the number of fourth grade school classes), using as sampling frame lists of schools provided by the Ministry of Education. From each school in the sample, two fourth grade classes were selected randomly. All children in the selected school classes were included in the study. On the whole, 51 schools were selected (102 classes), 62 in Athens, 18 in Thessaloniki and 22 in the other cities. We collected information on the adolescents' experiences and views about primary health care services using a structured questionnaire. We included questions on the usual context of their visits to primary care services, their perceived health status and usual problems, their opinion on several aspects of the organization of the services and the quality of their communication with health professionals. The questionnaire was administered to the students in the classroom during school hours. The maximum time needed to complete the questionnaire was $20 \mathrm{~min}$. Written consent from parents had been previously obtained. Approval of the research protocol has been obtained by the Bioethics Committee of the Institute of Child Health. We carried out data entry using Epidata (Epidata association, Denmark, version 3.1). Consistency and range checks were performed for data validation. The analysis was carried out using STATA (Stata Corporation, TX, USA, version 11). In the descriptive part of the analysis we calculated proportions and $95 \%$ confidence intervals adjusting for the sampling design implemented (stratification and cluster sampling). For testing the statistical significance of differences between proportions we used the chi square $\left(\chi^{2}\right)$ test.
We carried out multiple logistic regression to identify predictive factors independently associated with outcome variables. As outcome variables we used a) satisfaction from primary care visits and b) reporting communication problems with physician. Initial regression models were constructed including all variables for which the $\mathrm{p}$-value in the bivariate analysis was less than 0.1 . We removed variables one at a time from the initial models on the basis of significance testing $(p<0.1)$ with the likelihood ratio test. The adjusted risk ratios derived from binomial regression using all the variables of the final logistic regression models are presented.

\section{Results}

The questionnaire was completed by 2019 of the 2342 students selected (response rate $86.2 \%$ ). The mean age of students participating in the study was 15.1 years (standard deviation 0.5 years) and girls were 54\%; $92 \%$ were born in Greece. More than half of the children were living in Athens (59\%), about one fifth in Thessaloniki and one fifth in other cities (Table 1). Most students were living with both parents (85\%), divorce being the main reason of living with one parent.

\section{Context of health visits}

The vast majority of students $(80 \%)$ reported frequent visits to a physician, i.e., at least every six months. For 1340/1837 of the children (73\%) the visits were usually paid to a "generalist physician" (pediatrician or internist in ambulatory settings, according to the practice in Greece). Half of them reported that they always visited the same doctor. Nine in ten students usually visited a primary care ambulatory setting, while $4 \%$ usually visited a hospital outpatient clinic. Girls were more likely to visit a female physician than boys (Table 2). For half of the adolescents, the last

\begin{tabular}{|c|c|c|c|c|}
\hline & $\begin{array}{c}\text { Total } \\
n(\%)^{a} \\
(\mathrm{~N}=2019)\end{array}$ & $\begin{array}{c}\text { Boys } \\
n(\%)^{a} \\
(\mathrm{~N}=927)\end{array}$ & $\begin{array}{c}\text { Girls } \\
n(\%)^{\mathrm{a}} \\
(\mathrm{N}=1092)\end{array}$ & $P$ value \\
\hline $\begin{array}{l}\text { City } \\
\text { Athens } \\
\text { Thessaloniki } \\
\text { Other cities } \\
\text { Total }\end{array}$ & $\begin{array}{c}1191(59.0) \\
389(19.3) \\
439(21.7) \\
2019\end{array}$ & $\begin{array}{c}548(59.1) \\
183(19.7) \\
196(21.1) \\
927\end{array}$ & $\begin{array}{c}643(58.9) \\
206(18.9) \\
243(22.3) \\
1092\end{array}$ & 0.835 \\
\hline $\begin{array}{l}\text { Country of birth } \\
\text { Greece } \\
\text { Albania } \\
\text { Other } \\
\text { Total }\end{array}$ & $\begin{array}{c}1811(91.9) \\
80(4.1) \\
80(4.1) \\
1971\end{array}$ & $\begin{array}{c}832(93.0) \\
33(3.7) \\
30(3.4) \\
895\end{array}$ & $\begin{array}{c}972(91.0) \\
47(4.4) \\
50(4.6) \\
1076\end{array}$ & 0.172 \\
\hline $\begin{array}{l}\text { Father's age } \\
<45 \\
45-54 \\
\geq 55 \\
\text { Total }\end{array}$ & $\begin{array}{c}466(25.4) \\
1119(60.9) \\
253(13.8) \\
1838\end{array}$ & $\begin{array}{c}195(23.7) \\
510(61.9) \\
119(14.4) \\
824\end{array}$ & $\begin{array}{c}271(26.7) \\
609(60.1) \\
134(13.2) \\
1014\end{array}$ & 0.417 \\
\hline $\begin{array}{l}\text { Mother's age } \\
<45 \\
45-54 \\
\geq 55 \\
\text { Total }\end{array}$ & $\begin{array}{c}1118(59.8) \\
731(38.1) \\
38(2.0) \\
1869\end{array}$ & $\begin{array}{c}496(59.7) \\
315(37.9) \\
20(2.4) \\
831\end{array}$ & $\begin{array}{c}622(59.9) \\
398(38.3) \\
18(1.7) \\
1038\end{array}$ & 0.616 \\
\hline $\begin{array}{l}\text { Father's educational level } \\
\text { Lower secondary education } \\
\text { Upper secondary education } \\
\text { Tertiary education } \\
\text { Total }\end{array}$ & $\begin{array}{c}357(19.3) \\
579(31.4) \\
910(49.3) \\
1846\end{array}$ & $\begin{array}{c}170(20.2) \\
268(31.9) \\
403(47.9) \\
841\end{array}$ & $\begin{array}{c}187(18.6) \\
311(30.9) \\
507(50.4) \\
1005\end{array}$ & 0.496 \\
\hline $\begin{array}{l}\text { Mother's educational level } \\
\text { Lower secondary education } \\
\text { Upper secondary education } \\
\text { Tertiary education } \\
\text { Total }\end{array}$ & $\begin{array}{c}283(15.0) \\
717(38.0) \\
886(47.0) \\
1886\end{array}$ & $\begin{array}{c}126(14.6) \\
321(37.2) \\
416(48.2) \\
863\end{array}$ & $\begin{array}{c}157(15.3) \\
396(38.7) \\
470(45.9) \\
1023\end{array}$ & 0.643 \\
\hline
\end{tabular}

a: Percentages adjusted for stratification and cluster design

Table 1: Demographic characteristics of the sample adolescents. 
Citation: Xekalaki A, Papamichail D, Nikolaidou P, Papadimitriou A, Panagiotopoulos T (2017) Health Care Experiences and Needs of Adolescents in Urban Greece: Implications for Planning Appropriate Health Care. Health Care Current Reviews 5: 208. doi: 10.4172/2375-4273.1000208

Page 3 of 8

visit to a doctor was a well-child care visit or a preparticipation athletic evaluation. Only one in four reported illness as the cause of the last visit. The usual waiting time was $\leq 15 \mathrm{~min}$ for six in 10 students and for a similar proportion of students the usual time of consultation was $\leq 15$ min. Half of the children reported that visiting the doctor was usually a common decision of the parents and themselves, but three in 10 reported they had no say in the decision. When asked if they had ever visited the doctor alone, $47 \%$ replied affirmatively; boys were more likely to have visited a doctor on their own. About one in four adolescents reported having a health problem (girls $29 \%$, boys $18 \%$ ), although $90 \%$ stated that their health status was very good or good (Table 2).

\section{Communication problems}

Half of the students participating in the study reported having communication problems with the physician(s) they usually visit in the framework of primary care $(1017 / 2016,50 \%)$. Reasons of

\begin{tabular}{|c|c|c|c|c|}
\hline Questions & $\begin{array}{c}\text { Total } \\
\mathrm{n}\left(\% ; 95 \% \mathrm{Cl}^{\mathrm{a}}\right)^{\mathrm{b}} \\
(\mathrm{N}=\mathbf{2 0 1 9})\end{array}$ & $\begin{array}{c}\text { Boys } \\
\text { n }\left(\% ; 95 \% \mathrm{Cl}^{\mathrm{a}}\right)^{\mathrm{b}} \\
(\mathrm{N}=927)\end{array}$ & $\begin{array}{c}\text { Girls } \\
\text { n }\left(\% ; 95 \% \mathrm{Cl}^{\mathrm{a}}\right)^{\mathrm{b}} \\
(\mathrm{N}=1092)\end{array}$ & $P$ value \\
\hline $\begin{array}{l}\text { How often do you visit a doctor? } \\
\text { At least once in every six months } \\
\leq \text { once per year } \\
\text { Total }\end{array}$ & $\begin{array}{c}1609(80.0 ; 77.4-82.3) \\
403(20.0 ; 17.7-22.6) \\
2012\end{array}$ & $\begin{array}{c}736(79.8 ; 76.9-82.5) \\
186(20.2 ; 17.5-23.1) \\
922\end{array}$ & $\begin{array}{c}873(80.1 ; 76.6-83.2) \\
217(19.9 ; 16.8-23.4) \\
1090\end{array}$ & 0.887 \\
\hline $\begin{array}{l}\text { What was the cause for your last vis } \\
\text { doctor? } \\
\text { Well child visit }+ \text { /- vaccination } \\
\text { Sickness } \\
\text { Health assessment for sports } \\
\text { Other issue } \\
\text { Total }\end{array}$ & $\begin{array}{c}437(22.2 ; 20.2-24.4) 475 \\
(24.1 ; 21.6-26.8) 653(33.2 ; \\
30.3-36.3) 402(20.4 ; 18.5- \\
22.5) \\
1967\end{array}$ & $\begin{array}{c}170(19.0 ; 16.6-21.7) \\
204(22.8 ; 19.5-26.4) \\
331(37.0 ; 33.0-41.1) \\
190(21.2 ; 18.7-24.0) \\
895\end{array}$ & $\begin{array}{c}267(24.9 ; 22.0-28.0) \\
271(25.3 ; 21.9-29.0) \\
322(30.0 ; 26.4-33.9) \\
212(19.8 ; 16.8-23.1) \\
1072\end{array}$ & 0.006 \\
\hline $\begin{array}{l}\text { The doctor that you usually visit: } \\
\text { Is always the same person } \\
\text { Most of the times is the same person } \\
\text { Most of the times is a different person } \\
\text { Total }\end{array}$ & $\begin{array}{c}923(47.2 ; 45.2-49.3) 815 \\
(41.7 ; 39.5-43.9) \\
216(11.1 ; 9.3-13.1) \\
1954\end{array}$ & $\begin{array}{c}427(47.9 ; 44.9-50.8) \\
372(41.7 ; 38.9-44.5) \\
93(10.4 ; 8.2-13.2) \\
892\end{array}$ & $\begin{array}{c}496(46.7 ; 44.0-49.4) \\
443(41.7 ; 39.0-44.5) \\
123(11.6 ; 9.5-14.1) \\
1062\end{array}$ & 0.612 \\
\hline $\begin{array}{l}\text { What is the sex of the doctor that yo } \\
\text { usually visit? } \\
\text { Always a man } \\
\text { Always a woman } \\
\text { Most of the times a man } \\
\text { Most of the times a woman } \\
\text { Total }\end{array}$ & $\begin{array}{c}648(32.4 ; 30.2-34.7) 340 \\
(17.0 ; 15.2-19.1) 660(33.0 \\
31.0-35.1) 350(17.5 ; 15.5- \\
19.7) 1998\end{array}$ & $\begin{array}{c}341(37.2 ; 34.2-40.3) \\
144(15.7 ; 13.1-18.7) \\
296(32.3 ; 29.5-35.2) \\
136(14.8 ; 12.4-17.6) \\
917\end{array}$ & $\begin{array}{c}307(28.4 ; 25.6-31.4) \\
196(18.1 ; 15.6-20.9) \\
364(33.7 ; 31.0-36.4) \\
214(19.8 ; 17.2-22.7) \\
1081\end{array}$ & $<0.001$ \\
\hline $\begin{array}{l}\text { Who does normally decide that you } \\
\text { visit the doctor? } \\
\text { Parents } \\
\text { Myself } \\
\text { Parents and myself } \\
\text { Another person } \\
\text { Total }\end{array}$ & $\begin{array}{c}581(28.9 ; 26.6-31.4) \\
265(13.2 ; 11.8-14.7) \\
1135(56.5 ; 54.1-58.9) \\
28(1.4 ; 0.9-2.2) \\
2009\end{array}$ & $\begin{array}{c}270(29.3 ; 25.9-32.9) \\
148(16.1 ; 14.1-18.2) \\
485(52.7 ; 49.2-56.1) \\
18(2.0 ; 1.2-3.3) \\
921\end{array}$ & $\begin{array}{c}311(28.6 ; 25.9-31.5) \\
117(10.8 ; 9.0-12.8) \\
650(59.7 ; 56.6-62.8) \\
10(0.9 ; 0.4-1.9) \\
1088\end{array}$ & $<0.001$ \\
\hline $\begin{array}{l}\text { Have you ever visited the doctor alo } \\
\text { Yes } \\
\text { No } \\
\text { Total }\end{array}$ & $\begin{array}{c}907(46.6 ; 43.4-49.9) \\
1038(53.4 ; 50.1-56.6) \\
1945\end{array}$ & $\begin{array}{c}497(56.7 ; 52.6-60.8) \\
379(43.3 ; 39.2-47.4) \\
876\end{array}$ & $\begin{array}{c}410(38.4 ; 34.4-42.5) \\
659(61.6 ; 57.5-65.6) \\
1069\end{array}$ & $<0.001$ \\
\hline $\begin{array}{l}\text { What is the usual waiting time? } \\
\leq 15 \mathrm{~min} \\
>15 \mathrm{~min} \\
\text { Total }\end{array}$ & $\begin{array}{l}1271(63.3 ; 61.0-65.5) \\
738(36.7 ; 34.5-39.0) \\
2009\end{array}$ & $\begin{array}{l}597(64.8 ; 60.9-68.4) \\
325(35.2 ; 31.6-39.1) \\
922\end{array}$ & $\begin{array}{c}674(62.0 ; 58.7-65.2) \\
413(38.0 ; 34.8-41.3) \\
1087\end{array}$ & 0.303 \\
\hline $\begin{array}{l}\text { What is the usual consultation time? } \\
\leq 15 \min \\
>15 \min \\
\text { Total }\end{array}$ & $\begin{array}{c}1270(63.2 ; 61.0-65.4) \\
738(36.8 ; 34.6-39.0) \\
2008\end{array}$ & $\begin{array}{c}593(64.4 ; 61.2-67.4) \\
328(35.6 ; 32.6-38.8) \\
921\end{array}$ & $\begin{array}{c}677(62.3 ; 59.2-65.3) \\
410(37.7 ; 34.7-40.8) \\
1087\end{array}$ & 0.338 \\
\hline $\begin{array}{l}\text { Do you have a health problem? } \\
\text { Yes } \\
\text { No } \\
\text { Total }\end{array}$ & $\begin{array}{c}476(23.7 ; 21.7-25.9) \\
1532(76.3 ; 74.1-78.3) \\
2008\end{array}$ & $\begin{array}{c}166(18.0 ; 15.3-21.0) \\
756(82.0 ; 79.0-84.7) \\
922\end{array}$ & $\begin{array}{c}310(28.5 ; 25.4-31.9) \\
776(71.5 ; 68.1-74.6) \\
1086\end{array}$ & $<0.001$ \\
\hline $\begin{array}{l}\text { How would you describe your health } \\
\text { Very good } \\
\text { Good } \\
\text { Moderate } \\
\text { Bad } \\
\text { Total }\end{array}$ & $\begin{array}{c}936(46.9 ; 44.3-49.4) \\
863(43.2 ; 40.4-46.1) \\
174(8.7 ; 7.5-10.1) \\
24(1.2 ; 0.7-1.9) \\
1997\end{array}$ & $\begin{array}{c}500(54.3 ; 50.8-57.9) \\
350(38.0 ; 34.9-41.3) \\
61(6.6 ; 5.1-8.5) \\
9(1.0 ; 0.5-2.0) \\
920\end{array}$ & $\begin{array}{c}436(40.5 ; 36.9-44.2) \\
513(47.6 ; 43.7-51.5) \\
113(10.5 ; 8.8-12.4) \\
15(1.4 ; 0.8-2.3) \\
1077\end{array}$ & $<0.001$ \\
\hline
\end{tabular}

a: $95 \%$ Confidence Interval

b: Percentages adjusted for stratification and cluster design

Table 2: Context of adolescents' primary health care. 
Citation: Xekalaki A, Papamichail D, Nikolaidou P, Papadimitriou A, Panagiotopoulos T (2017) Health Care Experiences and Needs of Adolescents in Urban Greece: Implications for Planning Appropriate Health Care. Health Care Current Reviews 5: 208. doi: 10.4172/2375-4273.1000208

communication problems reported by these adolescents included: a/ organizational problems, e.g. not having their own doctor $(25 \%)$, inadequate consultation time (17\%); b/ privacy and confidentiality issues, e.g. not having the opportunity to be alone with the doctor (36\%), lack of confidence that their discussion with the doctor will not be disclosed to their parents (46\%); c/ lack of understanding their perspective, e.g. the doctor "is not interested in my problems" (15\%), he/she "does not treat me with respect" (13\%), he/she "criticizes the way I dress or behave
(13\%), "we do not speak the same language" (20\%). Of note, about one third of the adolescents who reported communication problems stated as a reason that doctors are "authoritarian and absolute" (Table 3).

Interestingly, among participants reporting communication problems with physicians, students from Thessaloniki were more likely to affirm that physicians do not treat them with respect ( $23 \%$ vs. $13 \%$ for students from Athens and $11 \%$ for those from other cities, $\mathrm{p}=0.017$ ),

\begin{tabular}{|c|c|c|c|c|}
\hline Question & $\begin{array}{c}\text { Total } \\
\mathrm{n}\left(\% ; 95 \% \mathrm{Cl}^{\mathrm{a}}\right)^{\mathrm{b}} \\
(\mathrm{N}=1017)\end{array}$ & $\begin{array}{c}\text { Boys } \\
\mathrm{n}\left(\% ; 95 \% \mathrm{Cl}^{\mathrm{a}}\right)^{\mathrm{b}} \\
(\mathrm{N}=451)\end{array}$ & $\begin{array}{c}\text { Girls } \\
\text { n }\left(\% ; 95 \% \mathrm{Cl}^{\mathrm{a}}\right)^{\mathrm{b}} \\
(\mathrm{N}=566)\end{array}$ & $P$ value \\
\hline $\begin{array}{l}\text { Time with the doctor is insufficient } \\
\text { Yes } \\
\text { No } \\
\text { Total }\end{array}$ & $\begin{array}{c}176(17.3 ; 14.7-20.4) \\
840(82.7 ; 79.6-85.3) \\
1016\end{array}$ & $\begin{array}{c}99(22.0 ; 17.7-26.9) \\
352(78.0 ; 73.1-82.3) \\
451\end{array}$ & $\begin{array}{c}77(13.6 ; 11.1-16.7) \\
488(86.4 ; 83.3-88.9) \\
565\end{array}$ & $<0.001$ \\
\hline $\begin{array}{l}\text { No confidentiality (disclosure to parents) } \\
\text { Yes } \\
\text { No } \\
\text { Total }\end{array}$ & $\begin{array}{l}466(45.9 ; 42.5-49.3) \\
550(54.1 ; 50.7-57.5) \\
1016\end{array}$ & $\begin{array}{l}204(45.2 ; 40.5-50.1) \\
247(54.8 ; 49.9-59.5) \\
451\end{array}$ & $\begin{array}{c}262(46.4 ; 42.3-50.5) \\
303(53.6 ; 49.5-57.7) \\
565\end{array}$ & 0.692 \\
\hline $\begin{array}{l}\text { I am never alone with the doctor } \\
\text { Yes } \\
\text { No } \\
\text { Total }\end{array}$ & $\begin{array}{c}370(36.4 ; 33.1-39.8) \\
646(63.6 ; 60.2-66.9) \\
1016\end{array}$ & $\begin{array}{c}157(34.8 ; 30.2-39.7) \\
294(65.2 ; 60.3-69.8) \\
451\end{array}$ & $\begin{array}{c}213(37.7 ; 33.2-42.4) \\
352(62.3 ; 57.6-66.8) \\
565\end{array}$ & 0.381 \\
\hline $\begin{array}{l}\text { The doctor is not interested in my problems } \\
\text { Yes } \\
\text { No } \\
\text { Total }\end{array}$ & $\begin{array}{c}152(15.0 ; 12.5-17.9) 864(85.0 \\
82.1-87.5) 1016\end{array}$ & $\begin{array}{c}92(20.4 ; 16.8-24.6) 359 \text { (79.6; } \\
75.4-83.2) 451\end{array}$ & $\begin{array}{c}60(10.6 ; 8.2-13.7) \\
505(89.4 ; 86.3-91.8) \\
565\end{array}$ & $<0.001$ \\
\hline $\begin{array}{l}\text { I don't believe that I can tell my problems to } \\
\text { the doctor } \\
\text { Yes } \\
\text { No } \\
\text { Total }\end{array}$ & $\begin{array}{c}513(50.5 ; 47.0-53.9) 503(49.5 \\
46.1-53.0) 1016\end{array}$ & $\begin{array}{c}221(49.0 ; 44.4-53.6) 230(51.0 \\
46.4-55.6) 451\end{array}$ & $\begin{array}{c}292(51.7 ; 47.2-56.1) 273(48.3 \\
43.9-52.8) 565\end{array}$ & 0.369 \\
\hline $\begin{array}{l}\text { The doctor doesn't treat me with respect } \\
\text { Yes } \\
\text { No } \\
\text { Total }\end{array}$ & $\begin{array}{c}136(13.4 ; 11.2-15.9) 880(86.6 \\
84.1-88.8) 1016\end{array}$ & $\begin{array}{c}76(16.9 ; 13.2-21.2) 375(83.1 \\
78.8-86.8) 451\end{array}$ & $\begin{array}{c}60(10.6 ; 8.2-13.7) \\
505(89.4 ; 86.3-91.8) \\
565\end{array}$ & 0.012 \\
\hline $\begin{array}{l}\text { The doctors are criticizing the way I dress and } \\
\text { behave } \\
\text { Yes } \\
\text { No } \\
\text { Total }\end{array}$ & $\begin{array}{c}127(12.5 ; 10.4-14.9) 889(87.5 \\
85.1-89.6) \\
1016\end{array}$ & $\begin{array}{c}72(16.0 ; 12.4-20.2) 379(84.0 \\
79.8-87.6) \\
451\end{array}$ & $\begin{array}{c}55(9.7 ; 7.5-12.5) \\
510(90.3 ; 87.5-92.5) \\
565\end{array}$ & 0.006 \\
\hline $\begin{array}{l}\text { Doctors don't speak the same language with } \\
\text { me. } \\
\text { Yes } \\
\text { No } \\
\text { Total }\end{array}$ & $\begin{array}{c}202(19.9 ; 17.3-22.7) \\
814(80.1 ; 77.3-82.7) \\
1016\end{array}$ & $\begin{array}{c}103(22.8 ; 19.3-26.8) \\
348(77.2 ; 73.2-80.7) \\
451\end{array}$ & $\begin{array}{c}99(17.5 ; 14.6-20.9) \\
466(82.5 ; 79.1-85.4) \\
565\end{array}$ & 0.022 \\
\hline $\begin{array}{l}\text { Doctors are authoritarian and absolute. } \\
\text { Yes } \\
\text { No } \\
\text { Total }\end{array}$ & $\begin{array}{l}281(27.7 ; 24.4-31.1) \\
735(72.3 ; 68.9-75.6) \\
1016\end{array}$ & $\begin{array}{c}128(28.4 ; 24.1-33.1) \\
323(71.6 ; 66.9-75.9) \\
451\end{array}$ & $\begin{array}{c}153(27.4 ; 23.3-31.3) \\
412(72.9 ; 68.7-76.7) \\
565\end{array}$ & 0.618 \\
\hline $\begin{array}{l}\text { Doctors think that I am still a little child and I } \\
\text { don't understand things } \\
\text { Yes } \\
\text { No } \\
\text { Total }\end{array}$ & $\begin{array}{c}368(36.2 ; 33.2-39.3) 648(63.8 \\
60.7-66.8) \\
1016\end{array}$ & $\begin{array}{c}170(37.7 ; 32.9-42.7) 281(62.3 \\
57.3-67.1) \\
451\end{array}$ & $\begin{array}{c}198(35.0 ; 31.5-38.8) 367 \text { (65.0; } \\
61.2-68.5) \\
565\end{array}$ & 0.375 \\
\hline $\begin{array}{l}\text { I don't have my own doctor } \\
\text { Yes, I don't have my own doctor } \\
\text { No, I have a doctor of my own. } \\
\text { Total }\end{array}$ & $\begin{array}{c}266(26.2 ; 23.2-29.4) \\
750(73.8 ; 70.6-76.8) \\
1016\end{array}$ & $\begin{array}{c}118(26.2 ; 22.1-30.7) \\
333(73.8 ; 69.3-77.9) \\
451\end{array}$ & $\begin{array}{c}148(26.2 ; 20.2-30.6) \\
417(73.8 ; 69.4-77.8) \\
565\end{array}$ & 0.992 \\
\hline
\end{tabular}

a: $95 \%$ Confidence Interval

b: Percentages adjusted for stratification and cluster design

Table 3: Communication problems reported. 
Citation: Xekalaki A, Papamichail D, Nikolaidou P, Papadimitriou A, Panagiotopoulos T (2017) Health Care Experiences and Needs of Adolescents in Urban Greece: Implications for Planning Appropriate Health Care. Health Care Current Reviews 5: 208. doi: 10.4172/2375-4273.1000208

Page 5 of 8

\begin{tabular}{|c|c|c|c|c|}
\hline Question & $\begin{array}{c}\text { Total } \\
\text { n }\left(\% ; 95 \% \mathrm{Cl}^{\mathrm{a}}\right)^{\mathrm{b}} \\
(\mathrm{N}=2019)\end{array}$ & $\begin{array}{c}\text { Boys } \\
\mathrm{n}\left(\% ; 95 \% \mathrm{Cl}^{\mathrm{a}}\right)^{\mathrm{b}} \\
(\mathrm{N}=927)\end{array}$ & $\begin{array}{c}\text { Girls } \\
\mathrm{n}\left(\% ; 95 \% \mathrm{Cl}^{\mathrm{a}}\right)^{\mathrm{b}} \\
(\mathrm{N}=1092)\end{array}$ & $P$ value \\
\hline $\begin{array}{l}\text { I would like to visit the doct } \\
\text { sometimes } \\
\text { I agree/probably agree } \\
\text { I disagree/probably disagree } \\
\text { Total }\end{array}$ & $\begin{array}{c}1329(73.5 ; 71.4-75.6) 478(26.5 \\
24.4-28.6) \\
1807\end{array}$ & $\begin{array}{c}665(79.9 ; 76.9-82.7) \\
167(20.1 ; 17.3-23.1) \\
832\end{array}$ & $\begin{array}{c}664(68.1 ; 64.7-71.3) \\
311(31.9 ; 28.7-35.3) \\
975\end{array}$ & $<0.001$ \\
\hline $\begin{array}{l}\text { I would like some issues to } \\
\text { private and confidential } \\
\text { I agree/probably agree } \\
\text { I disagree/probably disagree } \\
\text { Total }\end{array}$ & $\begin{array}{c}1530(80.5 ; 78.9-81.9) \\
371(19.5 ; 18.1-21.1) \\
1901\end{array}$ & $\begin{array}{c}683(79.6 ; 77.2-81.8) \\
175(20.4 ; 18.2-22.8) \\
858\end{array}$ & $\begin{array}{c}847(81.2 ; 78.9-83.3) \\
196(18.8 ; 16.7-21.1) \\
1043\end{array}$ & 0.332 \\
\hline $\begin{array}{l}\text { Is the sex of the doctor imp } \\
\text { you? } \\
\text { Yes } \\
\text { No } \\
\text { Total }\end{array}$ & $\begin{array}{c}738(36.7 ; 34.5-38.9) 1273(63.3 \\
61.1-65.5) \\
2011\end{array}$ & $\begin{array}{c}214(23.2 ; 20.2-26.5) \\
709(76.8 ; 73.5-79.8) \\
923\end{array}$ & $\begin{array}{c}524(48.2 ; 44.8-51.5) 564(51.8 \\
48.5-55.2) 1088\end{array}$ & $<0.001$ \\
\hline $\begin{array}{l}\text { What sex do you prefer the } \\
\text { would be? } \\
\text { Man } \\
\text { Woman } \\
\text { I don't care } \\
\text { Total }\end{array}$ & $\begin{array}{c}243(12.1 ; 10.9-13.5) 743(37.0 ; \\
34.8-39.3) 1020(50.8 ; 48.7-53.0) \\
2006\end{array}$ & $\begin{array}{c}183(19.9 ; 17.7-22.3) \\
155(16.9 ; 14.2-19.9) \\
581(63.2 ; 59.8-66.5) \\
919\end{array}$ & $\begin{array}{c}60(5.5 ; 4.1-7.4) \\
588(54.1 ; 50.9-57.2) 439(40.4 ; \\
37.1-43.7) 1087\end{array}$ & $<0.001$ \\
\hline $\begin{array}{l}\text { My parents would not allow } \\
\text { alone with the doctor } \\
\text { I agree/probably agree } \\
\text { I disagree/probably disagree } \\
\text { Total }\end{array}$ & $\begin{array}{c}255(13.3 ; 11.8-14.8) \\
1668(86.7 ; 85.2-88.2) \\
1923\end{array}$ & $\begin{array}{c}125(14.1 ; 11.8-16.7) \\
761(85.9 ; 83.3-88.2) \\
886\end{array}$ & $\begin{array}{c}130(12.5 ; 10.6-14.7) \\
907(87.5 ; 85.3-89.4) \\
1037\end{array}$ & 0.345 \\
\hline $\begin{array}{l}\text { The waiting room makes me } \\
\text { uncomfortable } \\
\text { I agree/probably agree } \\
\text { I disagree/probably disagree } \\
\text { Total }\end{array}$ & $\begin{array}{c}584(29.8 ; 27.2-32.5) \\
1379(70.2 ; 67.5-72.8) \\
1963\end{array}$ & $\begin{array}{c}256(28.4 ; 24.4-32.7) \\
645(71.6 ; 67.3-75.6) \\
901\end{array}$ & $\begin{array}{c}328(30.9 ; 27.7-34.2) \\
734(69.1 ; 65.8-72.3) \\
1062\end{array}$ & 0.343 \\
\hline $\begin{array}{l}\text { The waiting time is usually } t \\
\text { I agree/probably agree } \\
\text { I disagree/probably disagree } \\
\text { Total }\end{array}$ & $\begin{array}{c}935(48.0 ; 44.8-51.2) \\
1012(52.0 ; 48.8-55.2) \\
1947\end{array}$ & $\begin{array}{c}400(44.5 ; 40.2-48.9) \\
499(55.5 ; 51.1-59.8) \\
899\end{array}$ & $\begin{array}{c}535(51.0 ; 47.1-54.9) \\
513(49.0 ; 45.1-52.9) \\
1048\end{array}$ & 0.016 \\
\hline $\begin{array}{l}\text { I would like more time to dis } \\
\text { the doctor } \\
\text { I agree/probably agree } \\
\text { I disagree/probably disagree } \\
\text { Total }\end{array}$ & $\begin{array}{c}603(32.2 ; 29.3-35.3) \\
1267(67.8 ; 64.7-70.7) \\
1870\end{array}$ & $\begin{array}{c}270(31.5 ; 27.9-35.4) \\
586(68.5 ; 64.6-72.1) \\
856\end{array}$ & $\begin{array}{c}333(32.8 ; 29.1-36.8) \\
681(67.2 ; 63.2-70.9) \\
1014\end{array}$ & 0.203 \\
\hline $\begin{array}{l}\text { I feel uncomfortable during } \\
\text { examination } \\
\text { I agree/probably agree } \\
\text { I disagree/probably disagree } \\
\text { Total }\end{array}$ & $\begin{array}{c}650(33.4 ; 30.7-36.2) \\
1297(66.6 ; 63.8-69.3) \\
1947\end{array}$ & $\begin{array}{c}176(19.6 ; 16.1-23.8) \\
720(80.4 ; 76.2-83.9) \\
896\end{array}$ & $\begin{array}{c}474(45.1 ; 41.8-48.4) \\
577(54.9 ; 51.6-58.2) \\
1051\end{array}$ & $<0.001$ \\
\hline $\begin{array}{l}\text { I feel uncomfortable in askir } \\
\text { questions. } \\
\text { I agree/probably agree } \\
\text { I disagree/probably disagree } \\
\text { Total }\end{array}$ & $\begin{array}{c}568(29.1 ; 26.9-31.5) \\
1381(70.9 ; 68.5-73.1) \\
1949\end{array}$ & $\begin{array}{c}211(23.8 ; 20.1-27.8) \\
677(76.2 ; 72.2-79.9) \\
888\end{array}$ & $\begin{array}{c}357(33.6 ; 30.7-36.7) \\
704(66.4 ; 63.3-69.3) \\
1061\end{array}$ & 0.005 \\
\hline $\begin{array}{l}\text { Doctors don't explain what } \\
\text { with me and what I should d } \\
\text { I agree/probably agree } \\
\text { I disagree/probably disagree } \\
\text { Total }\end{array}$ & $\begin{array}{c}351(17.9 ; 16.0-20.0) \\
1609(82.1 ; 80.0-84.0) \\
1960\end{array}$ & $\begin{array}{c}152(17.1 ; 14.6-19.8) \\
739(82.9 ; 80.2-85.4) \\
891\end{array}$ & $\begin{array}{c}199(18.6 ; 16.2-21.4) \\
870(81.4 ; 78.6-83.8) \\
1069\end{array}$ & 0.341 \\
\hline $\begin{array}{l}\text { Are you satisfied with the h} \\
\text { that you have experienced? } \\
\text { Very much } \\
\text { Sufficiently } \\
\text { Moderately } \\
\text { Not at all } \\
\text { Total }\end{array}$ & $\begin{array}{c}481(24.2 ; 22.0-26.4) 1071(53.8 \\
51.5-56.1) 391(19.6 ; 17.7-21.8) \\
48(2.4 ; 1.9-3.1) \\
1991\end{array}$ & $\begin{array}{c}263(28.9 ; 25.8-32.1) \\
475(52.1 ; 48.6-55.6) \\
144(18.8 ; 13.0-19.0) \\
29(3.2 ; 2.2-4.5) \\
911\end{array}$ & $\begin{array}{c}218(20.2 ; 17.4-23.2) \\
596(55.2 ; 52.4-58.0) \\
247(22.9 ; 20.3-25.7) \\
19(1.8 ; 1.2-2.5) \\
1080\end{array}$ & $<0.001$ \\
\hline
\end{tabular}

a: $95 \%$ Confidence Interval

b: Percentages adjusted for stratification and cluster design

Table 4: Adolescents' views on health care provision. 
don't "speak the same language" (30\% vs. $18 \%$ and $22 \%$ respectively, $\mathrm{p}=0.025)$ or don't give them enough time ( $30 \%$ vs. $16 \%$ and $17 \%$ respectively, $\mathrm{p}=0.009)$, while students from "other cities" were more likely to raise confidentiality issues ( $54 \%$ vs. $43 \%$ for participants from Athens and $45 \%$ for those from Thessaloniki, $\mathrm{p}=0.017)$.

\section{Views and suggestions of adolescents}

The large majority of adolescents in the study sample stated that they would like to have the opportunity to visit the doctor on their own (boys $72 \%$, girls $61 \%$ ) and to have a private and confidential discussion with him/her (boys $86 \%$, girls $87 \%$ ); most of the children (87\%) believed that their parents would not object to confidential consultations. The gender of the physician is important for about one third of the students (girls $48 \%$, boys $23 \%$ ) and girls tend to prefer a female doctor (54\%). More consultation time available for discussing important matters was suggested by one third of study participants and one in five stated that doctors do not provide adequate explanations. The majority of children (78\%) affirmed that, on the whole, they were satisfied (very much or sufficiently) with the health care they get (Table 4).

\section{Multivariable analysis}

According to the multivariable analysis staying in Thessaloniki or in other cities and the higher educational level of the father is positively correlated with better communication with the doctor. In contrast adolescent smoking is increasing the chance of communication problems. Furthermore, higher educational level of the mother and living with both parents is associated with more satisfaction from health care; adolescents who smoke are less likely to be satisfied with health care they receive (Table 5).

\section{Discussion}

We carried out a cross-sectional study in a random sample of 15 year old adolescents living in large cities in Greece to investigate their experiences and views on health care services and make policy recommendations. About half of the adolescents participating in the study reported having communication problems with the physician they usually visit. This is a significant problem with far reaching implications, which has been attributed by study participants to several specific conditions. These include organization of care in a way that does not provide them "their doctor" (i.e., continuation of care), inadequate consultation time, lack of sensitivity from the part of physicians to "understand them", as well as aspects of care and physician behavior that do not ensure privacy and make them feel uncertain about confidentiality. Communicating effectively with adolescents requires knowledge of this unique stage of development and an ability to understand the social context of health behaviors in adolescence, which are different from those of children or adults [22]. Research has shown that pediatricians can view adolescents as difficult or demanding patients with a special agenda of issues they cannot always understand or handle $[23,24]$. Lack of training can be an important barrier in delivering effective adolescent health care and formal training is suggested for physicians and other health care professionals [23-27]. In a review, Melville (1989) argued that teenagers frequently have a hidden agenda behind the presenting physical symptoms, which, unless trained, a health professional may find it difficult to unlock [28]. Teaching of consultation and communication skills has been shown to be an effective means of improving doctors' skills in consulting, and specific training in consultation skills for pediatric encounters has been advocated $[29,30]$. It has been shown that health professionals have credibility among adolescents and the advice they give may have a positive impact on health behavior [31]. Furthermore, studies have reported that a familiar trusted person can improve communication and adolescents are more willing to discuss topics that have been previously discussed [31]. Time available for consultation is an important parameter of increased quality in health care of adolescents, as has been consistently shown $[23,24,27,32,33]$. Confidentiality and privacy have been also shown to be critical for high quality health care for adolescents and research has shown that adolescents who forgo health care due to confidentiality concerns are particularly vulnerable

\begin{tabular}{|c|c|c|c|c|}
\hline & $\begin{array}{c}\text { Satisfaction from } \\
\text { health care experience }\end{array}$ & & $\begin{array}{l}\text { Communication problems } \\
\text { with physician }\end{array}$ & \\
\hline Predictive factor & $\begin{array}{c}\text { Adjusted } \mathrm{RR}^{\mathrm{a}}\left(95 \% \mathrm{Cl}^{\mathrm{b}}\right) \\
(\mathrm{N}=1846)\end{array}$ & $P$ value & $\begin{array}{c}\text { Adjusted } \mathrm{RR}^{\mathrm{a}}\left(95 \% \mathrm{Cl}^{\mathrm{b}}\right) \\
(\mathrm{N}=1831)\end{array}$ & $P$ value \\
\hline $\begin{array}{l}\text { Sex } \\
\text { Boy } \\
\text { Girl }\end{array}$ & $\begin{array}{c}\text { Ref. } \\
0.93(0.89-0.98)\end{array}$ & 0.010 & NA $\ddagger$ & \\
\hline $\begin{array}{l}\text { City of residence } \\
\text { Athens } \\
\text { Thessaloniki } \\
\text { Other cities }\end{array}$ & $\begin{array}{c}\text { Ref. } \\
1.00(0.93-1.07) \\
1.09(1.03-1.16)\end{array}$ & $\begin{array}{l}0.948 \\
0.005\end{array}$ & $\begin{array}{c}\text { Ref. } \\
0.47(0.35-0.63) \\
0.92(0.78-1.09)\end{array}$ & $\begin{array}{c}<0.001 \\
0.344\end{array}$ \\
\hline $\begin{array}{l}\text { Father's educational level } \\
\text { Medium educational level } \\
\text { High educational level }^{\mathrm{d}}\end{array}$ & $N A^{e}$ & & $\begin{array}{c}\text { Ref. } \\
0.89(0.82-0.97)\end{array}$ & 0.009 \\
\hline $\begin{array}{l}\text { Mother's educational level } \\
\text { Medium educational level } \\
\text { High educational level }\end{array}$ & $\begin{array}{c}\text { Ref. } \\
1.06(1.01-1.10)\end{array}$ & 0.008 & $N A^{e}$ & \\
\hline $\begin{array}{l}\text { Living with both parents } \\
\text { No } \\
\text { Yes }\end{array}$ & $\begin{array}{c}\text { Ref. } \\
1.09(1.00-1.19)\end{array}$ & 0.033 & $N A^{e}$ & \\
\hline $\begin{array}{l}\text { Adolescent smoking } \\
\text { No } \\
\text { Yes }\end{array}$ & $\begin{array}{c}\text { Ref. } \\
0.91(0.84-0.97)\end{array}$ & 0.005 & $\begin{array}{c}\text { Ref. } \\
1.18(1.07-1.31)\end{array}$ & 0.001 \\
\hline
\end{tabular}

a: Adjusted risk ratio

b: $95 \%$ Confidence Interval

c: Medium educational level: up to higher secondary school level graduates

d: High educational level: tertiary education graduates

e: Non-applicable (not in the final model of multivarable analysis)

Table 5: Predictive factors for adolescents being satisfied from health care and for reporting communication problems: final model of logistic regression analysis. 
and in need of health services [34,35]. Many adolescents want to speak alone with their doctors about sensitive health topics but often refrain due to lack of opportunity for private discussion [34]. The issue of adolescent confidentiality in health care presents challenges for health care providers related to family, medical, ethical, legal, social and administrative processes [36]. Parents are usually willing to allow their daughters and sons consult doctors alone, as long as confidentiality policy and limitations are explained to them [33]. The importance of parents' agreeing to a private discussion is related to the legal aspects of consent, competence and confidentiality regarding adolescents under the age of 18 in the context of the legal framework of any given country $[18,35,37]$. Even though parental consent may be granted, uncertainty over ethical and legal rights and responsibilities may lead professionals to refuse to see adolescents on their own for fear of incurring parental wrath or even legal action $[37,38]$. Due to the importance of these issues, various health care organizations have issued relevant guidelines [33]. Of note, none of the specialties involved in primary care of adolescents in Greece have any systematic training in adolescent medicine, while this is not a recognized subspecialty in Greece. Moreover, adolescent medicine is not part of the core curriculum at pre-graduate level. In the present study, we found that $90 \%$ of adolescents' visits to a doctor are paid to a "generalist physician" (pediatrician or internist in ambulatory settings), which is in line with previous reports [29]. In most cases (75\%), these visits were carried out in the context of "well child visits". Furthermore, $80 \%$ of adolescents reported that they visit a doctor at least once in every six months (Table 2). This is consistent with research findings in other developed countries, which indicate that $70-90 \%$ of adolescents contact primary health services at least once a year [2,31]. Therefore, present practice provides opportunities for consultations with adolescents, which, if appropriately oriented, can address critical problems of this stage of development. It is noteworthy that despite adolescents' critical views about the primary care services they get, as discussed above, the majority of them (78\%) reported that they were very much or sufficiently satisfied with the health care they had experienced (Table 4). This possibly reflects low expectations from physicians and health care encounters, particularly regarding advice on sensitive issues.

\section{Strengths and Limitations}

A notable strength of the present study is that it provides detailed and quantified information on adolescents' views regarding their health care and their encounters with primary care physicians in the context of large cities in Greece; these can serve as a basis for a comprehensive plan to reorganize health care services for adolescents in Greece, particularly at a time when restructuring of primary care services is under way [39]. Furthermore, our study provides information on groups of adolescents whose needs should be particularly the focus of attention by services and physicians, as they report more communication problems with doctors and/or reduced satisfaction from health services (e.g. children with parents of low or medium educational level, children of one-parent families, adolescents who smoke (Table 5).

An important limitation of our study is the fact that the study population was adolescents from large cities $(60,000$ inhabitants or more). Therefore, our conclusions refer to this type of populations in Greece and cannot be directly extrapolated to adolescents from smaller areas. Moreover, our findings are based on views of adolescents through a detailed questionnaire; although these give an important insight, views of parents and physicians as well as morbidity based measures of health care needs are also important, but have not been included in the present study. In conclusion, adolescents in this study pointed out that they need doctors who are aware of their way of thinking and problems, and can communicate effectively with them, they require more consultation time, continuity of care, respect of confidentiality and opportunities for consultations in privacy. In Greece, the reform of primary health care which is in progress and modifications planned for curricula of health professional education and specialization should take seriously into account the specifics of adolescent health care needs.

\section{References}

1. Viner RM, Barker M (2005) Young people's health: The need for action. BMJ 330: 901-903.

2. Tylee A, Haller DM, Graham T (2007) Youth-friendly primary-care services: How are we doing and what more needs to be done? Lancet 369: 1565-1573.

3. Patton GC, Coffey C, Cappa C (2012) Health of the world's adolescents: A synthesis of internationally comparable data. Lancet 379:1665-1675.

4. Gore FM, Bloem PJ, Patton GC (2011) Global burden of disease in young people aged 10-24 years: A systematic analysis. Lancet 377: 2093-2102.

5. Macfarlane A, McPherson A (1995) Primary health care and adolescence. BM 311: 825-826.

6. Patton GC, Viner R (2007) Pubertal transitions in health. Lancet 369: 11301139 .

7. Kleinert S (2007) Adolescent health: An opportunity not to be missed. Lance 369: 1057-1058.

8. Blum RW (2009) Young people: Not as healthy as they seem. Lancet 374 853-854

9. World Health Organization (2017) Current trends in adolescent health in Europe.

10. Viner RM, Coffey C, Mathers C (2011) 50 year mortality trends in children and young people: A study of 50 low-income, middle-income and high-income countries. Lancet 377: 1162-1174.

11. Due P, Lynch J, Holstein B, Modvig J (2003) Socioeconomic health inequalities among a nationally representative sample of Danish adolescents: The role of different types of social relations. J Epidemiol Community Health 57: 692-698.

12. Swedish National Institute of Public Health (2017) Social health inequalities in Swedish children and adolescents - A systematic review.

13. Petanidou D, Giannakopoulos G, Tzavara C (2012) Identifying the sociodemographic determinants of subjective health complaints in a crosssectional study of Greek adolescents. Ann Gen Psychiatry 11: 17

14. Currie C, Nic Gabhainn S, Godeau E, Roberts C, Smith R, et al. (2008) Inequalities in young people's health: HBSC international report from the 2005/06 Survey. Health Policy for Children and Adolescents, No. 5, WHO Regional Office for Europe, Copenhagen, Denmark.

15. Patton GC, Coffey C, Sawyer SM (2009) Global patterns of mortality in young people: A systematic analysis of population health data. Lancet 374: 881-192.

16. World Health Organization (2017) Adolescent friendly health services: An agenda for change.

17. Ambresin AE, Bennett K, Patton GC (2013) Assessment of youth-friendly health care: A systematic review of indicators drawn from young people's perspectives. J Adolesc Health 52: 670-681.

18. Kang M, Bernard D, Booth M (2003) Access to primary care for Australian young people: Service provider perspectives. Br J Gen Pract 53: 947-952.

19. Patton GC, Viner R (2007) Pubertal transitions in health. Lancet 369: 11301139 .

20. European Commission (2013) Report on health inequalities in the European Union. Brussels.

21. Kentikelenis A, Papanicolas I (2012) Economic crisis, austerity and the Greek public health system. Viewpoint. Eur J Public Health 22: 4-5.

22. McPherson A (2005) Adolescents in primary care. BMJ 330: 465-467.

23. Jacobson L, Richardson G, Parry-Langdon N, Donovan C (2001) How do teenagers and primary healthcare providers view each other? An overview of key themes. Br J Gen Pract 51: 811-816. 
Citation: Xekalaki A, Papamichail D, Nikolaidou P, Papadimitriou A, Panagiotopoulos T (2017) Health Care Experiences and Needs of Adolescents in Urban Greece: Implications for Planning Appropriate Health Care. Health Care Current Reviews 5: 208. doi: 10.4172/2375-4273.1000208

Page 8 of 8

24. Akinbami LJ, Gandhi H, Cheng TL (2003) Availability of adolescent health services and confidentiality in primary care practices. Pediatrics 111: 394 401.

25. Anderson J E, Lowen C (2010) Connecting youth with health services: Systematic review. Can Fam Physician 56: 778-784.

26. Veit FC, Sanci LA, Coffey CM (1996) Barriers to effective primary health care for adolescents. Med J Aust 165: 131-133.

27. Kang M, Bernard D, Booth M (2003) Access to primary care for Australian young people: Service provider perspectives. Br J Gen Pract 53: 947-952.

28. Melville AWT (1986) Caring for adolescents. Fam Pract 6: 245-246.

29. Cahill P, Papageorgiou A (2007) Triadic communication in the primary care paediatric consultation: A review of the literature. Br J Gen Pract 57: 904-911.

30. Sanci L A, Coffey CMM, Veit FCM (2000) Evaluation of the effectiveness of an educational intervention for general practitioners in adolescent health care: Randomised controlled trial. BMJ 320: 224-230.

31. Walker Z, Townsend J, Oakley L (2002) Health promotion for adolescents in primary care: Randomized controlled trial. BMJ 325:524.
32. Jacobson LD, Wilkinson C, Owen PA (1994) Is the potential of teenage consultations being missed? A study of consultation times in primary care. Fam Pract 11: 296-299.

33. McKee MD, Rubin E, Campos G, O'Sullivan LF (2011) Challenges of providing confidential care to adolescents in urban primary care: Clinician perspectives. Ann Fam Med. 9: 37-43.

34. Lehrer JA, Pantell R, Tebb K, Shafer MA (2007) Forgone health care among U.S. adolescents: Associations between risk characteristics and confidentiality concern. J Adolesc Health 40: 218-226.

35. Ford C, Sigman G (2004) Confidential health care for adolescents: Position paper of the society of adolescent medicine. J Adolesc Health 35: 160-167.

36. Kokkevi A, Stavrou M, Kanavou E, Fotiou A (2014) The Repercussions of the economic recession in Greece on adolescents and their families. UNICEF, pp: $1-18$

37. Larcher V (2005) Consent, competence and confidentiality. BMJ 330: 353-356.

38. Bravender T, Price CN, English A (2004) Primary care providers' willingness to see unaccompanied adolescents. J Adolesc Health 34: 30-36.

39. Primary health care reform bill [Greek] (2017) Ministry of Health. 